\title{
Human Control of Leader-Based Swarms
}

\author{
Phillip Walker, Saman Amirpour Amraii, and \\ Michael Lewis \\ School of Information Sciences \\ University of Pittsburgh \\ Pittsburgh, PA 15213, USA \\ pmw19epitt.edu, samirpourdacm.org, \\ mlasis.pitt.edu
}

\author{
Nilanjan Chakraborty and Katia Sycara \\ Robotics Institute \\ Carnegie Mellon University \\ Pittsburgh, PA 15213, USA \\ nilanjanecs.cmu.edu, katiaecs.cmu.edu
}

\begin{abstract}
As swarms are used in increasingly more complex scenarios, further investigation is needed to determine how to give human operators the best tools to properly influence the swarm after deployment. Previous research has focused on relaying influence from the operator to the swarm, either by broadcasting commands to the entire swarm or by influencing the swarm through the teleoperation of a leader. While these methods each have their different applications, there has been a lack of research into how the influence should be propagated through the swarm in leader-based methods. This paper focuses on two simple methods of information propagation-flooding and consensus-and compares the ability of operators to maneuver the swarm to goal points using each, both with and without sensing error. Flooding involves each robot explicitly matching the speed and direction of the leader (or matching the speed and direction of the first neighboring robot that has already done so), and consensus involves each robot matching the average speed and direction of all the neighbors it senses. We discover that the flooding method is significantly more effective, yet the consensus method has some advantages at lower speeds, and in terms of overall connectivity and cohesion of the swarm.
\end{abstract}

\section{INTRODUCTION}

In the near future, swarms of robots may replace humans and single robots for a number of common tasks. Because swarms are made up of numerous robots that operate under scalable, distributed algorithms, they can cover more area more robustly than a single robot or teams of independent robots. This makes them suitable for jobs such as exploration and foraging [1], [2], [3], construction [4], [5], and fire fighting or HAZMAT situations [6], [7]. Indeed, in the recent years, we have seen swarms move from a theoretical possibility to systems implemented on real robots in laboratory settings, such as those in [8], [9], [10].

While there are some cases where swarms might act with full autonomy, many tasks require some sort of coordination between a human operator and the swarm. For instance, if the operator is using a swarm of unmanned vehicles (UVs) to survey a large outdoor area, they may want to alter several details of the UVs' operations, such as which areas should be explored first, what routes they should take, and how

This research has been sponsored in part by AFOSR FA955008-10356 and ONR Grant N0001409-10680.

978-1-4799-0652-9/13/\$31.00 2013 IEEE closely they should move with respect to each other. Because swarm algorithms are designed to work with large numbers of robots, individual teleoperation of each swarm member is not feasible. Instead, the operator usually controls the swarm as a whole, as if it were one entity.

\section{A. Approaches to Swarm Control}

Researchers have taken numerous approaches to injecting human influence into a robot swarm. In [9], the authors create a user interface whereby operators can use video game controllers to move around subgroups of the swarm, or take control of individuals when necessary. This idea of controlling the swarm as a single group is continued in [2] and [3], where the authors broadcast global heading commands to the swarm, and then the robots perform consensus algorithms to overcome odometry error and flock in a common direction. Other work has expanded on this by taking inspiration from the literature on levels of automation (LOAs) and applying that to swarm control. In [11], the researchers design an autonomy spectrum, based on the original 10-point scale in [12], to guide the design on human-swarm systems and investigate how humans perceive a swarm.

Broadcasting is usually used when the human has to send commands intermittently to the swarm system. For transmitting continuous commands to affect the swarm system, or when it is not feasible for each robot to have the ability to communicate directly with the human, leader-based swarm control methods have been proposed. In leader-based swarm control, the operator directly controls a single swarm member, or a small subset, and that influence propagates to the rest of the swarm depending on the underlying control laws the swarm members use. This approach has been investigated extensively by Goodrich [13], who used leaders with attractive virtual forces, and predators with repulsive forces, to steer a swarm around a simulated environment. There the authors found that leader-based control, as opposed to predator-based control, was more manageable for human operators, and that swarms operating under physics-based (physicomimetic) control laws [14] gave similar performance to those operating under biologically-inspired control laws. Leader-based swarm control frameworks have also been investigated for possible applications in space research and 
exploration [15].

\section{B. Our Contribution and Hypotheses}

The underlying task that we will use is an outdoor survey task where the goal of the operator is to guide the swarm to different target regions that arise dynamically. The operator can continuously give a velocity to the leader robot, after which information propagates according to two methodsflooding and consensus. Flooding involves propagating that velocity information in a manner similar to breadth-first search (BFS), whereby robots search for neighbors that have received the goal speed and heading, and attempt to match the first of these "privileged" neighbors they see. Consensus involves each robot averaging the headings of all of its neighbors, and moving toward that average. A theoretical study and comparison of the two methods in simulated stationary wireless sensor networks can be found in [16]. In this paper, we perform user experiments to study the effect of each propagation method on the ability of operators to steer a flocking swarm to designated goal regions. We also investigate the effect of the information propagation methods on different measures of cohesiveness of the swarm, e.g., mean connectivity and diameter. In practice, the swarm members may have some estimation errors about the velocities of their neighbors due to sensing error. Therefore, we further study the combined effect of estimation error and information propagation method on the ability of the operators to control the swarm.

We hypothesize that the flooding method will give better control overall, and thus result in a higher number of goal regions reached. This is due to the fact that with flooding, the user's intended direction and speed for the swarm is passed directly from the leader to neighboring robots, thus flooding the network in the number of consensus rounds no greater than the diameter of the sensing graph of the swarm. However, we believe that the consensus method will result in a more cohesive swarm-in terms of connectivity and swarm diameter-and be more robust against sensing error, due to the fact that each robot is averaging its intended speed and heading across all neighbors, instead of just the nearest neighbor who has matched the leader, as is the case in the flooding method.

\section{TASK DESCRIPTION}

Our study investigates the ability of human operators to control a flocking swarm of robots in an open environment by teleoperating a single leader via a continuous velocity (i.e. heading and speed) command. The main task for the users is to survey a given area by guiding the swarm to goal regions, which appear dynamically in the environment. A goal region appears at a random position only after another goal region has been visited by the swarm. Thus, the number of goal regions visited by the operator is a natural measure of his or her performance. As stated earlier, we investigate the differences between two methods of propagating the heading and speed command from the leader to the rest of the swarm. In addition, we investigate the effect of sensing error of the robots on the ability of the operator to control the swarm. The participants completed both the flooding and consensus propagation methods, each with and without sensing error (in total, four conditions).

\section{A. The Environment}

We use an open 100x100 meter environment, and Stage v.3.2.2 [17] to simulate 20 P2AT robots for the study. The robot controllers and user interface are implemented using the Robot Operating System (ROS) [18]. Each robot is equipped with a simulated neighbor sensor to determine the speed and heading of neighboring robots within 4 meters.

In the conditions with sensing error, simulated noise sampled from the Gaussian distribution $\mathcal{N}(0,0.2)$ meters is added to the ground truth $x$ and $y$ coordinates of the sensed robot. Similarly, simulated noise sampled from the Gaussian distribution $\mathcal{N}\left(0, \frac{\pi}{3}\right)$ is added to the ground truth heading, $\theta$, of the robot.

In each of the four conditions, the swarm of robots is initialized randomly in a 10x10 meter box, centered around the origin of the environment, each with a random starting orientation. The leader robot always begins on the origin, but with a random orientation.

\section{B. Human Influence}

The main goal of the human operator is to steer the swarm to goal regions shown as blue circles in the environment (Figure 1). Once over half the swarm reaches the goal region, a new goal appears at a random position. No goal regions are given outside the central 50x50 meters of the environment. This is to ensure operators will not spend the majority of their time waiting for the swarm to traverse the entire width of the environment to reach a goal region. However, the environment is kept at $100 \times 100$ meters to ensure proper space to turn and maneuver the swarm, should the operator lose control.

To influence the swarm, the operator is given a virtual joystick with which to steer the leader robot around (Figure 1). This joystick allows the operator to set both the heading and speed of the leader. The other robots move according to local control laws, hereafter called the align, cohere, and repel laws, which are explained below. The vectors given by each of the following laws are unit vectors (scalar value of 1), as the velocity is determined solely by the propagation from the leader. Finally, the maximum velocity of the non-leader robots in the swarm is set at $0.75 \mathrm{~m} / \mathrm{s}$, which through preliminary testing gave the best balance of speed and controllability; however the leader is allowed to reach speeds of $1.5 \mathrm{~m} / \mathrm{s}$. This is to allow the operator to purposefully separate the leader from the rest of the swarm, in the event they want to collect disconnected members or move the leader around to another side of the swarm without having the entire swarm follow.

1) Alignment: The alignment vector is determined by the propagation method (flooding or consensus). For the flooding propagation condition, each robot determines if any of it's 

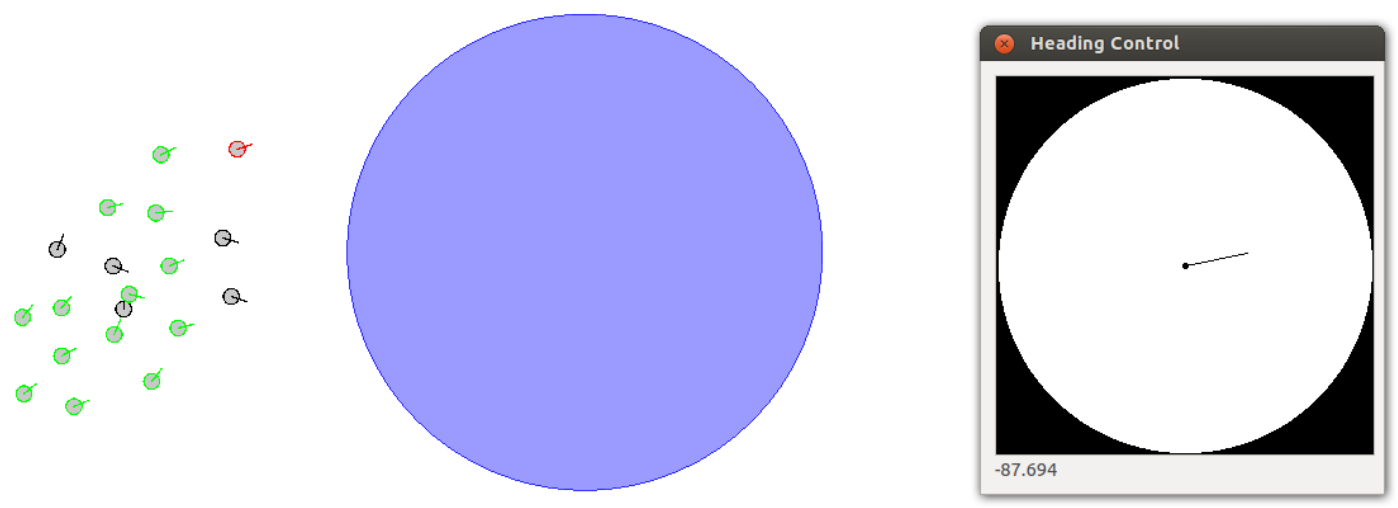

Fig. 1. The swarm of robots (left) is steered to the goal region (center) by the user teleoperating the leader robot (shown in red) using the virtual joystick (right). Robots that have detected the goal heading and speed from the leader (or an intervening neighbor) and are moving in that direction are shown in green.

neighbors are either the leader robot, or a privileged nonleader. A privileged robot is one which has sensed and matched the heading and speed of the leader or another privileged robot. Once a robot has sensed a privileged neighbor or leader, it will set its velocity and its alignment vector, $\left\langle x_{a}, y_{a}\right\rangle$, to match the velocity and heading of that neighbor in the robot's coordinate frame. Once the velocity and heading is matched (to within 15 degrees), the robot will enter privileged mode itself. Note that, in the conditions with sensing error, the neighbor heading being matched may be inaccurate, as it is based on the heading returned by the simulated neighbor sensor. In the event that neither the leader nor any privileged neighbors are detected, no alignment vector is used.

For the consensus propagation method, each robot averages the speed and heading of each neighbor it can sense, and then sets its speed and alignment vector $\left\langle x_{a}, y_{a}\right\rangle$ to that average speed and heading (again in the robot's coordinate frame). There is no special status given to the leader, nor are there any privileged robots in this case.

2) Cohesion and Repulsion: In addition to sensing neighbors' velocities, the robots also sense neighbors' positions to maintain swarm cohesion and avoid inter-robot collisions by using cohesion and repulsion laws that allow the swarm to flock together. The cohesion vector $\left\langle x_{c}, y_{c}\right\rangle$ is determined by summing the bearings to all neighboring robots relative to the given robot that are outside the cohesion threshold of 3.0 meters. The repulsion vector $\left\langle x_{r}, y_{r}\right\rangle$ is determined by summing the negative bearings to all neighboring robots relative to the given robot that are inside the repulsion threshold of 1.5 meters. All three of the vectors above are then summed to give the instantaneous motion direction, according to the equation below.

$$
\left\langle x_{g}, y_{g}\right\rangle=\alpha\left\langle x_{a}, y_{a}\right\rangle+\beta\left\langle x_{c}, y_{c}\right\rangle+\gamma\left\langle x_{r}, y_{r}\right\rangle
$$

Here, $\alpha, \beta$, and $\gamma$ represent constants to give different weights to each of the vectors. For this study, the parameters of $\alpha=1.5, \beta=1.0$, and $\gamma=1.0$ gave the best balance between the three vector components.

\section{Experimental Design}

Eighteen participants (14 men and 4 women) from the Pittsburgh, USA region participated in the study. Each participant received 3 minutes of training on each of the four conditions (flooding with and without error, and consensus with and without error). Following the training period, each participant completed the four conditions in a random order. Each condition counted as one trial, and lasted 10 minutes. At the conclusion of each trial, the participants were given a NASA-TLX workload questionnaire [19] to assess the workload of the trial.

\section{RESULTS}

The main measure of success for participants is the number of goal regions reached over the course of the 10 minutes for a condition. There was a significant difference between each of the four conditions for the number of goal regions reached $(F=72.45, p<.001$, see Figure 2). Participants were most successful in the flooding without error condition, where they reached an average of 13.78 goal regions. This was significantly more than the flooding with error condition ( $M=9.33, t=3.49, p=.001)$. Furthermore, participants were significantly more successful in reaching the goal regions in the flooding $(M=11.56)$ than the consensus ( $M=1.97)$ conditions overall $(t=12.26, p<.001)$. Within the consensus conditions, goals reached in the consensus without error condition ( $M=2.61)$ was significantly higher than in consensus with error $(M=1.33, t=2.46, p=$ $.021)$.

These findings are also confirmed by the results of the NASA-TLX workload questionnaire each participant took at the conclusion of each trial. Those results show that workload score differed significantly across conditions $(F=26.18$, $p<.001)$. Results between each individual condition are shown in I. Further investigation showed that workload was higher in the consensus $(M=69.78)$ than the flooding conditions $(M=39.24, t=7.84, p<.001)$, and higher in the error $(M=60.05)$ than the non-error conditions $(M=48.97, t=2.14, p=.039)$. 


\begin{tabular}{|l|l|l|l|l|}
\hline & 1 & 2 & 3 & 4 \\
\hline $1(M=31.81)$ & - & .009 & $<.001$ & $<.001$ \\
$2(M=46.67)$ & .009 & - & $<.001$ & $<.001$ \\
$3(M=66.13)$ & $<.001$ & $<.001$ & - & .164 \\
$4(M=73.43)$ & $<.001$ & $<.001$ & .164 & - \\
\hline
\end{tabular}

TABLE I

TABLE COMPARING NASA-TLX SCORES BETWEEN THE FOUR CONDITIONS OF THE STUDY. CONDITION NUMBERINGS ARE AS FOLLOWS: 1 = flooding without error, 2 = flooding with error, $3=$ consensus without error, AND $4=$ consensus with error

Next, we looked at connectivity and swarm diameter across each of the conditions. Connectivity was determined by taking the second eigenvalue of the sensing graph of the swarm - commonly called the Fiedler number-whereby a higher value indicates a more connected swarm. Swarm diameter is simply the furthest distance between any two robots in the largest connected component of the swarm, which gives a rough estimate of the spatial size of the swarm.

Investigating connectivity gave few significant results. Unsurprisingly, conditions without error showed significantly more connected swarms $(M=0.27)$ than conditions with error $(M=0.17, t=2.55, p=.013)$. Beyond that, the only two conditions that showed significant differences in connectivity were the consensus without error condition $(M=0.30)$ and the flooding with error condition $(M=$ $0.15)$, the former of which was significantly more connected than the latter $(t=2.34, p=.027)$. Connectivity between consensus $(M=0.24)$ and flooding $(M=0.20)$ were not significant $(t=0.92, p=.359)$.

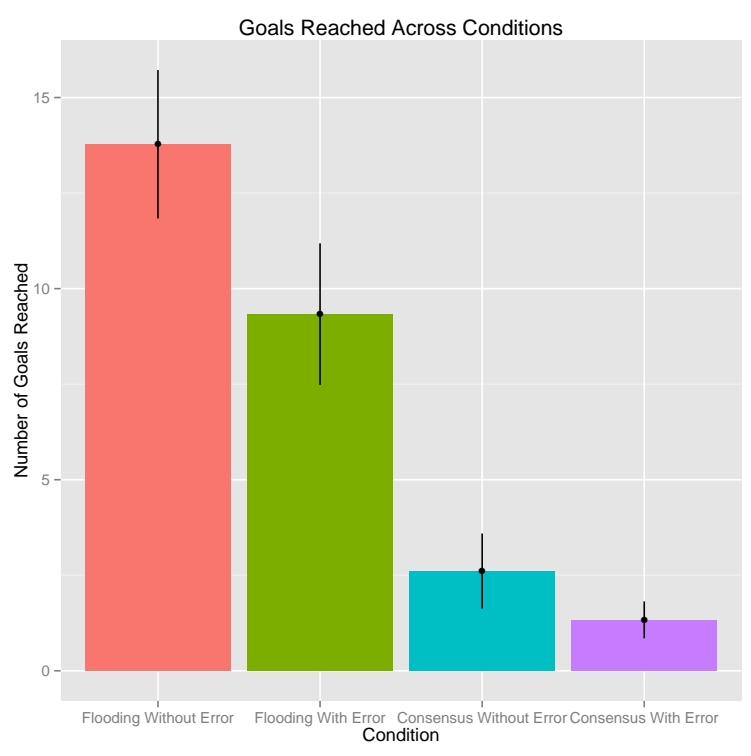

Fig. 2. The mean number of goals reached during each condition

There were significant differences between the conditions for swarm diameter, however $(F=20.48, p<.001$, see Figure 3). The diameter of the swarm was larger in the flooding conditions $(M=10.79 \mathrm{~m})$ than in the consensus

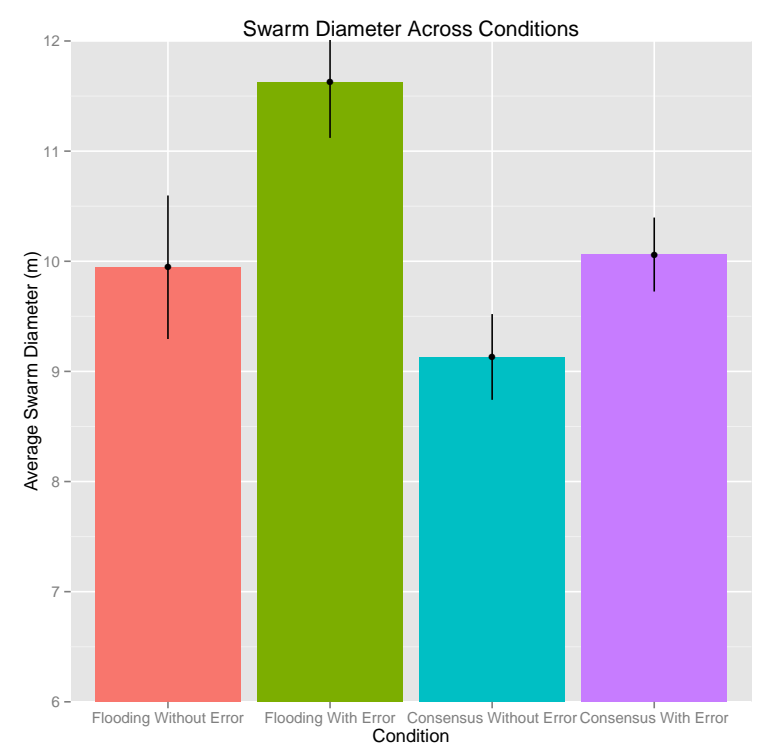

Fig. 3. The mean diameter of the swarm during each of the four conditions.

conditions $(M=9.60 \mathrm{~m}, t=4.26, p<.001)$. Similarly, the error conditions had a higher diameter $(M=10.84 \mathrm{~m})$ than the non-error conditions $(M=9.54 \mathrm{~m}, t=4.81, p<.001)$. Further information about significance between conditions can be found in Table II.

After analyzing the differences between conditions, we wanted to see what effect operator behaviors had on their ability to reach goals and on the swarm's overall cohesion. First, we investigated how the mean speed at which the operator maneuvered the leader affected these measures. A linear regression showed that mean leader speed has a positive correlation with goals reached for the flooding methods $\left(r^{2}=0.23, F=10.24, p=.003\right.$, see Figure 4), but a negative correlation for the consensus methods $\left(r^{2}=0.36, F=19.10, p<.001\right.$, see Figure 5). Despite this difference, mean leader speed had a negative correlation with mean connectivity $\left(r^{2}=0.43, F=53.09, p<.001\right)$ and swarm diameter $\left(r^{2}=0.21, F=18.43, p<.001\right)$ across all conditions. Further investigation, however, shows that the correlation between mean leader speed and diameter is driven by the error conditions. The error conditions combined show a negative correlation $\left(r^{2}=0.23, F=10.07, p=.003\right)$, yet the non-error conditions do not $\left(r^{2}=0.08, F=3.07\right.$, $p=.089$ ).

The other aspect of operator behavior we investigated was where the user positioned the leader on average relative to the rest of the swarm. By taking the absolute value of the difference between the average heading of the swarm, and the relative bearing from the swarm centroid to the leader, we determined that the closer the leader was to the front of the swarm, the better the operator was at reaching goal regions $\left(r^{2}=0.38, F=42.39, p<.001\right)$, a graphic demonstrating this fact this can be found in Figure 6. Relative bearing from the centroid to the leader had little effect on other measures, showing a marginal positive correlation with connectivity 
( $\left.r^{2}=0.05, F=3.54, p=.064\right)$, and no correlation with swarm diameter $\left(r^{2}=0.01, F=0.76, p=.388\right)$.

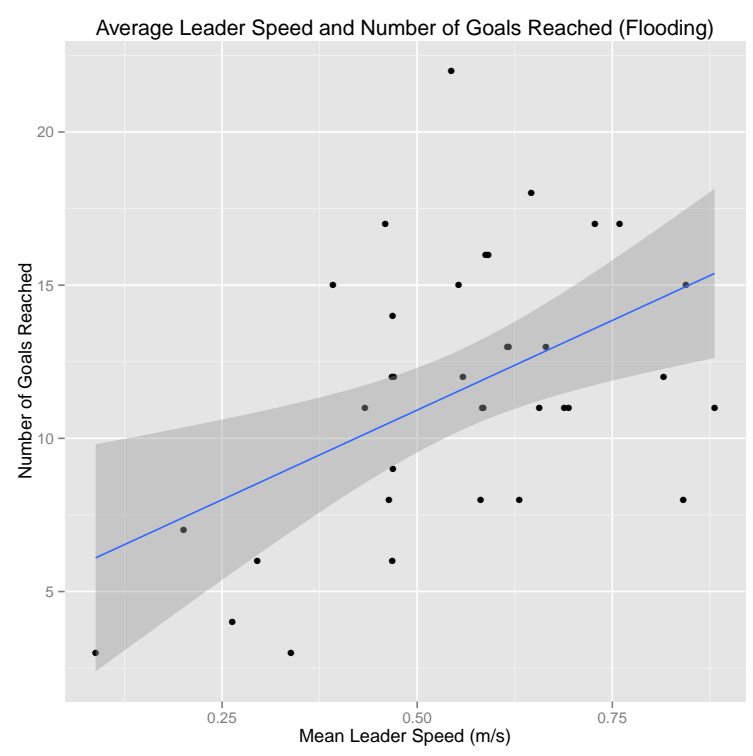

Fig. 4. Plot showing the positive relationship between mean leader speed and the number of goals reached for the flooding conditions. The gray band shows the error bounds for the linear model.

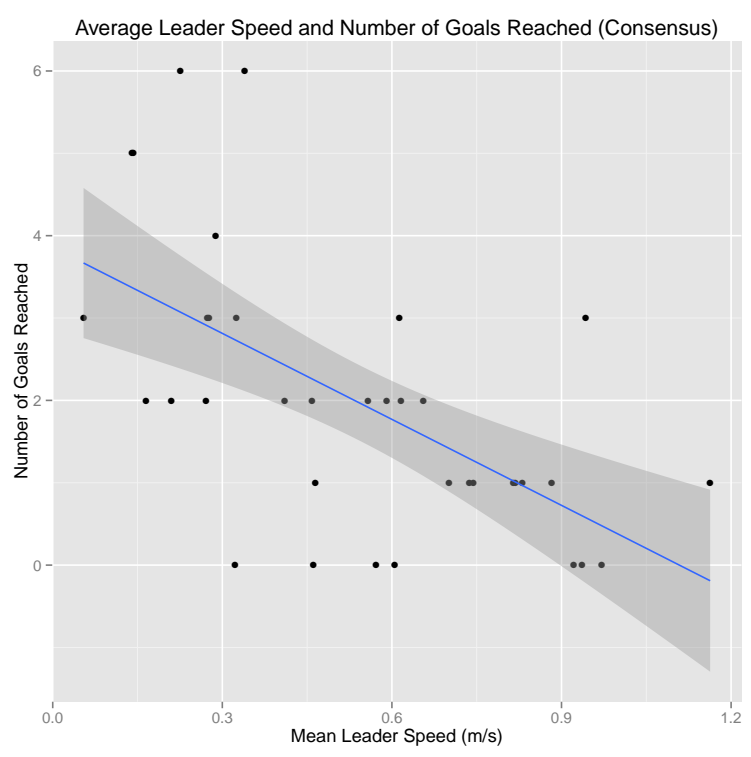

Fig. 5. Plot showing the negative relationship between mean leader speed and the number of goals reached for the consensus conditions. The gray band shows the error bounds for the linear model.

\section{DISCUSSION}

Overall, the results show that the flooding method of propagating information from the leader robot to the rest of the swarm is superior for maneuvering a swarm to target locations in an open environment. We believe that the primary reason for this is that with propagation by flooding, every member of the swarm has all the information

\begin{tabular}{|l|l|l|l|l|}
\hline & 1 & 2 & 3 & 4 \\
\hline $1(M=9.95)$ & - & $<.001$ & .032 & .743 \\
$2(M=11.62)$ & $<.001$ & - & $<.001$ & $<.001$ \\
$3(M=9.13)$ & .032 & $<.001$ & - & $<.001$ \\
$4(M=10.06)$ & .743 & $<.001$ & $<.001$ & - \\
\hline
\end{tabular}

TABLE II

TABLE COMPARING P-VALUES FOR SWARM DIAMETER BETWEEN THE FOUR CONDITIONS OF THE STUDY. CONDITION NUMBERINGS ARE THE SAME AS TABLE I

as quickly as possible, whereas with the consensus method, the propagation of operator influence takes much more time. In other words, the response latency of the swarm is much higher in consensus method compared to flooding. Hence, in the consensus methods, sharp (or fast) changes in direction made by the leader robot cause the rest of the swarm to become disconnected from the leader more easily, thereby making control of the swarm difficult. As the swarm grows in size, influencing the swarm via the consensus method will become even more difficult. However, this also means that smaller swarms could be much more manageable with averaging-based propagation. We initially thought that flooding could be at a disadvantage when it came to dealing with sensing error, and that using a consensus method instead could improve performance in these conditions. However, despite operators reaching fewer goals in the flooding with error condition, using the consensus method gave no clear reduction in the effect of error on the swarm's operation. Therefore, we would recommend the flooding method, or some similar method of immediate, breadth-first propagation over a slower averaging method in most cases of leader-based swarm control.

This does not mean that the consensus method is without merits. In the consensus conditions, the swarms were both more compact and had a more connected sensing graph. This could provide significant benefit if bandwidth between swarm members is at a premium, as a more highly connected network would allow for more messages to be passed through the swarm. Denser swarms could also be beneficial in cases of operation in obstacle-filled spaces, such as surveying the ocean floor or with small unmanned ground vehicles exploring urban and indoor environments. This is further supported by the finding that the consensus method was more successful at slower leader speeds (and thus slower speeds of the swarm overall), which are more common when working in tight spaces. In fact, at the lowest speeds (less than about $0.33 \mathrm{~m} / \mathrm{s}$ ), the consensus method was about as successful as the flooding method at allowing the operator to lead the swarm to goal regions (see Figures 4 and 5).

Finally, investigation of the relative position of the leader to the swarm centroid indicates that it is often better to "lead from ahead", meaning that the leader pulls the rest of the swarm along, rather than pushing from behind or the middle. This is initially counterintuitive, as leaders in the middle would give faster propagation for the flooding 


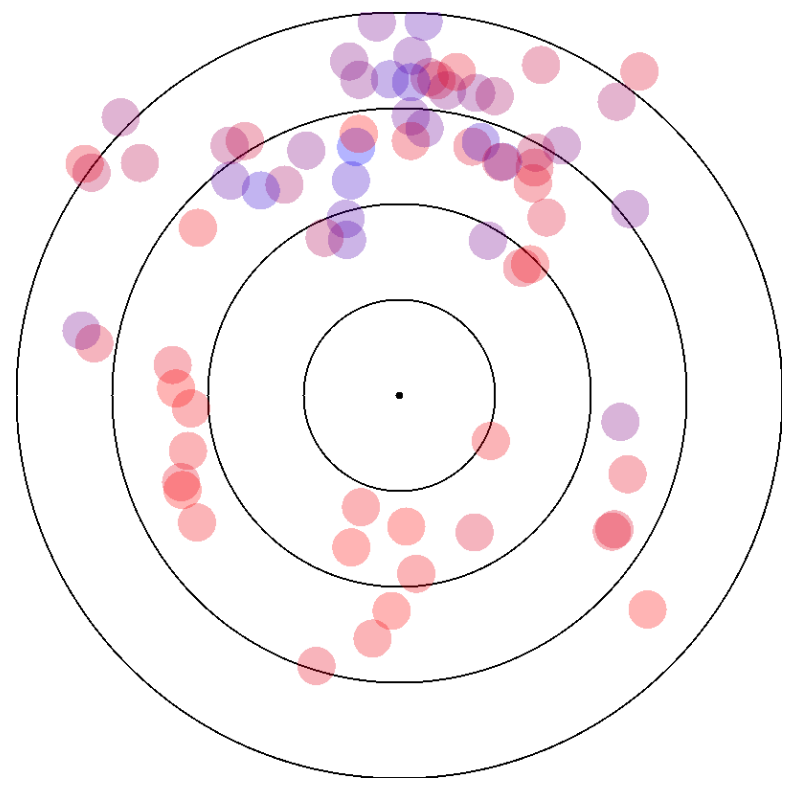

Fig. 6. Figure showing the relative number of goals reached by the mean position of the leader relative to the centroid of the swarm. The up direction indicates the average heading of the swarm. For instance, a circle directly above the center dot was, on average across that condition, directly in front of the swarm centroid in the direction the swarm was traveling. Bluer circles indicate a higher number of goals reached. Rings are placed around the centroid at 1 meter intervals.

method (as the total time to flood the network is simply the maximum number of hops in the sensing graph between the leader and any robot). However, results from [16] indicate that, for the consensus, or other averaging-based methods, a leader located slightly away from a central "hub" of the sensing graph is preferable to one in the center. In this case, leading from the front does not seem as unexpected, as it allows the leader to be slightly away from the center of the swarm. Still, further investigation is needed to determine why leading from the front is preferable to behind.

\section{CONCLUSIONS AND FUTURE WORKS}

In this paper we demonstrate the relative usefulness of two different propagation methods for a swarm influenced through a teleoperated leader. The two methods used were a flooding method, whereby information was propagated directly by hops through the sensing graph, and consensusa simple averaging-based method. Results show that the flooding method is more successful in a task where operators must quickly maneuver the swarm to goal regions in an open environment. This remains true even if the robots are affected by significant sensing error. However, averagingbased methods may still be useful in scenarios requiring a tightly compact swarm, or where the swarm must operate at lower speeds. Furthermore, in cases where communication bandwidth is limited, a tightly compact swarm would also be beneficial because it would allow for more connections between the robots, and thus theoretically a higher throughput for the network.

An important area for further analysis would be to determine if consensus-based propagation could be improved by using multiple leaders, and thus allowing the human to have a larger influence over the average of the swarm. This research could also investigate the proper proportion of the swarm that should be leaders, or be knowledgeable about the intention of the human operator, in a manner similar to [20] yet applied to human-swarm systems.

\section{REFERENCES}

[1] S. Bashyal and G. Venayagamoorthy, "Human swarm interaction for radiation source search and localization," in Swarm Intelligence Symposium, 2008. SIS 2008. IEEE. IEEE, 2008, pp. 1-8.

[2] P. Walker, S. Nunnally, M. Lewis, A. Kolling, N. Chakraborty, and K. Sycara, "Neglect benevolence in human control of swarms in the presence of latency," in Systems, Man, and Cybernetics (SMC), 2012 IEEE International Conference on. IEEE, 2012, pp. 3009-3014.

[3] S. Nunnally, P. Walker, A. Kolling, N. Chakraborty, M. Lewis, K. Sycara, and M. Goodrich, "Human influence of robotic swarms with bandwidth and localization issues," Systems, Man, and Cybernetics (SMC), 2012 IEEE International Conference on, pp. 333-338, 2012.

[4] C. Parker, H. Zhang, and C. Kube, "Blind bulldozing: multiple robot nest construction," in Intelligent Robots and Systems, 2003.(IROS 2003). Proceedings. 2003 IEEE/RSJ International Conference on, vol. 2. IEEE, 2003, pp. 2010-2015.

[5] Y. Meng and J. Gan, "A distributed swarm intelligence based algorithm for a cooperative multi-robot construction task," in Swarm Intelligence Symposium, 2008. SIS 2008. IEEE. IEEE, 2008, pp. 1-6.

[6] A. Naghsh, J. Gancet, A. Tanoto, and C. Roast, "Analysis and design of human-robot swarm interaction in firefighting," in Robot and Human Interactive Communication, 2008. RO-MAN 2008. The 17th IEEE International Symposium on. IEEE, 2008, pp. 255-260.

[7] D. Bruemmer, "A robotic swarm for spill finding and perimeter formation," DTIC Document, Tech. Rep., 2002.

[8] M. Dorigo, E. Tuci, R. Groß, V. Trianni, T. Labella, S. Nouyan, C. Ampatzis, J. Deneubourg, G. Baldassarre, S. Nolfi et al., "The swarm-bots project," Swarm Robotics, pp. 31-44, 2005.

[9] J. McLurkin, J. Smith, J. Frankel, D. Sotkowitz, D. Blau, and B. Schmidt, "Speaking swarmish: Human-robot interface design for large swarms of autonomous mobile robots," in Proc. of the AAAI Spring Symposium, 2006, pp. 72-75.

[10] A. Turgut, H. Çelikkanat, F. Gökçe, and E. Şahin, "Self-organized flocking in mobile robot swarms," Swarm Intelligence, vol. 2, no. 2 , pp. 97-120, 2008.

[11] G. Coppin and F. Legras, "Autonomy spectrum and performance perception issues in swarm supervisory control," Proceedings of the IEEE, no. 99, pp. 590-603, 2012.

[12] T. Sheridan and W. Verplank, "Human and computer control of undersea teleoperators," DTIC Document, Tech. Rep., 1978.

[13] M. Goodrich, B. Pendleton, P. Sujit, and J. Pinto, "Toward human interaction with bio-inspired robot teams," in Systems, Man, and Cybernetics (SMC), 2011 IEEE International Conference on. IEEE, 2011, pp. 2859-2864.

[14] W. Spears and D. Spears, Physicomimetics: Physics-Based Swarm Intelligence. Springer-Verlag New York Inc, 2012.

[15] C. Rouff, M. Hinchey, W. Truszkowski, and J. Rash, "Verifying large numbers of cooperating adaptive agents," in Parallel and Distributed Systems, 2005. Proceedings. 11th International Conference on, vol. 1. IEEE, 2005, pp. 391-397.

[16] S. Amirpour Amraii, N. Chakraborty, and M. Lewis, "Studying direct and indirect human influence on consensus in swarms," in 2012 AAAI Fall Symposium Series, 2012.

[17] B. Gerkey, R. Vaughan, and A. Howard, "The player/stage project: Tools for multi-robot and distributed sensor systems," in Proceedings of the 11th international conference on advanced robotics. Portugal, 2003, pp. 317-323.

[18] M. Quigley, K. Conley, B. Gerkey, J. Faust, T. Foote, J. Leibs, R. Wheeler, and A. Ng, "Ros: an open-source robot operating system," in ICRA Workshop on Open Source Software, vol. 3, no. 3.2, 2009.

[19] S. G. Hart and L. E. Staveland, "Development of nasa-tlx (task load index): Results of empirical and theoretical research," Human mental workload, vol. 1, no. 3, pp. 139-183, 1988.

[20] I. D. Couzin, J. Krause, N. R. Franks, and S. A. Levin, "Effective leadership and decision-making in animal groups on the move,' Nature, vol. 433, no. 7025, pp. 513-516, 2005. 